\title{
Estrategia segura y efectiva para el manejo de la neumonía adquirida en la comunidad
}

A Controlled Trial of a Critical Pathway for treatment of Community-acquired Pneumonia.
Marrie TJ; Lau CY; Wheeler SL; Wong CJ; Vandervoort M; Feagan BG; for the CAPITAL Study Investigators. JAMA 2000; 283: 749-75.

\section{Objetivo}

La neumonía adquirida en la comunidad (NAC) es una entidad que se maneja en forma muy heterogénea, lo que genera grandes variaciones en la utilización de recursos. La falta de estrategias comunes para manejarla podría ser la explicación de este fenómeno.

El objetivo del presente trabajo fue determinar si la utilización de un camino crítico mejora la eficiencia y mantiene la seguridad y bienestar de los pacientes en el manejo de NAC.

\section{Diseño}

Estudio clínico en el que se asignó a los hospitales a dos estrategias diferentes en forma aleatoria (aleatorización en cluster o racimos*) con un seguimiento de los pacientes durante seis semanas.

\section{Pacientes}

Se incluyeron 1743 pacientes que concurrieron a departamentos de emergencia pertenecientes a hospitales escuela y comunitarios de Canadá entre enero y julio de 1998.

\section{Intervención}

Diez hospitales fueron aleatorizados al manejo habitual de la NAC y nueve, a implementar un camino crítico consistente en una decisión de internación guiada por una regla de predicción clínica (pneumonia severity index: PSI) y uso de levofloxacina.

\section{Medición de resultados principales}

Se evaluó la efectividad mediante la calidad de vida de los pacientes con el cuestionario (SF36) a las seis semanas, considerándose significativa una diferencia de tres o más puntos en dicho puntaje. Se midió la utilización de recursos a través de número de días cama por paciente manejado (DCPM), una medida que tiene en cuenta la tasa de internación y la estadía hospitalaria. Se midieron como puntos finales secundarios la mortalidad y los eventos adversos.

\section{Resultados}

No hubo diferencias en la calidad de vida, complicaciones o mortalidad entre los grupos. El límite de una cola para la diferencia en el puntaje SF36 fue de 2.4 puntos. El grupo en el que se utilizó el camino crítico mostró una reducción de 1.7 días (18\%) en el DCPM Los pacientes manejados con el camino crítico recibieron con mayor frecuencia tratamiento con una sola clase de antibióticos y menos días de tratamiento endovenoso.

\section{Conclusiones}

El manejo de la NAC siquiendo un camino crítico redujo la utilización de recursos sin alterar el resultado clínico ni la satisfacción de los pacientes.

\section{COMENTARIO}

Las guías de práctica clínica y los caminos críticos son documentos que están ganando su lugar en la práctica cotidiana. Artículos cómo el que motiva este comentario agregan información que no es frecuente encontrar sobre la utilización en un ambiente real de las guías midiendo resultados clínicamente relevantes y apropiados.

Los autores plantean claramente la pregunta sobre la efectividad de la guía de práctica y eligieron un estudio clínico controlado tomando como unidad de análisis los hospitales y randomizándolos a implementar la guía o a manejar de manera convencional a los pacientes con NAC. Los puntos finales elegidos son interesantes: la medida de resultados primaria fue el SF36, evaluándose también la mortalidad, el reingreso äl hospital y las complicaciones de la neumonía como medidas de para comparar la eficacia y seguridad de ambas estrategias. La medida de utilización de recursos es un indicador indirecto (o "subrogante") del costo de un episodio de neumonía que tiene en cuenta la tasa de admisión de neumonías y la estadía promedio de cada institución. Cómo bien señalan los autores, la mayor parte del costo en neumonía está generado por la internación de pacientes en el hospital. Dado que uno de los objetivos de la guía es reducir la tasa de admisión y la estadía, la medida utilizada es apropiada. Evaluar la efectividad de una intervención clínica obliga a que las poblaciones estén bien caracterizadas y si bien una aleatorización adecuada puede asegurarnos que todos los posibles factores confundidores de la asociación entre utilización de la guía de práctica y los puntos finales propuestos han sido balanceados, el medir estos potenciales factores agrega sin duda solidez a los resultados del estudio. Un punto que puede generar controversia es que el grupo que no recibió la intervención experimental (grupo control de este estudio) pudo haber en realidad ser tratado de manera similar al grupo intervención ya que varios conceptos importantes en el desarrollo de la guía ya habían sido publicados con anterioridad a la realización de este estudio ${ }^{1-2}$. Esto podría haber conducido a que el grupo que no utilizaba la intervención pudiera en realidad Referencias

1. a generic severity of illness measure for patients with community acquired Fine MJ; Hanusa BH; Lave JR et al: Comparison of a disease specific and pneumonia . J gen int med 10:359-368, 1995 2. File TM; Segreti J; Dunbar L et al: a multicenter, randomized study comparing the efficacy and safety of intravenous and /or oral levofloxacin versus ceftriaxone and/or cefuroxime acetil in treat ment of adults with community-acquiered pneumonia. Antimicrob. Agents Chemoter 41: 1965-1972, 1997.

3. Mandell LA: Antibiotic Therapy for community-acquired pneumonia. Clinics in Chest Medicine 20(3):589-598, 1999

4. Luna CM; Efron ED; Schiavi E; Gené RJ; Famiglietti A; Jo lly EC; Niederman MS, or el grupo de estudios de las NAC: Neumonía adquirida en la comunidad en adultos. Guía de práctica clínica para la Argentina. Medicina (Buenos Aires) 1997; 57:343-355

5. Palmer CS, Zhan C, Elixhauser A, et al: Economic assessment of the community-acquired pneumonia intervention trial employing levofloxacin. Clin Ther 22(2):250-264, 2000 estar utilizando similares estrategias terapéuticas y, por lo tanto, alcanzado resultados similares al grupo experimental en los puntos finales clínicos. Un dato del reporte que aleja esta posibilidad es que los pacientes en los hospitales que usaban la guía tenían más probabilidad de ser tratados con una clase sola de antibióticos ( $27 \%$ vs $64 \%, p<0.001)$; y, por otra parte, que aún siendo parecidos los grupos en relación a su tratamiento, la utilización de la guía se asoció a un favorable efecto en la utilización de los recursos. La guía permitió obtener buenos resultados especialmente en los grupos de menor severidad, siendo más modestos en los pacientes de mayor severidad. Los primeros son, precisamente, los grupos de presentación más frecuente y en los que es esperado que exista mayor heterogeneidad en el manejo clínico. Otra inquietud que surge al leer el trabajo es si la levofloxacina es una elección racional como tratamiento de la neumonía adquirida en la comunidad. Una reciente revisión narrativa sobre antibioticoterapia en neumonías sostiene que en adultos que no tienen riesgo de tener neumococos resistentes a la penicilina, las nuevas quinolonas parecen no ser drogas de primera elección ${ }^{3}$. Las guías locales tampoco las recomiendan ${ }^{4}$. Sin embargo, es interesante una reciente evaluación económica de este mismo estudio donde se evalúa el costo global de la estrategia que usa levofloxacina comparada con la estrategia convencional que encontró un ahorro de entre u\$s 457 a u\$\$994 por paciente 5 . Esta favorable evaluación económica, empero, debe contrastarse con el riesgo de generar resistencia a esta novedosa clase de antibióticos. Para concluir, podemos decir que el manejo de la NAC en base a una guía de práctica clínica es beneficioso desde el punto de vista de uso de recursos y que se asocia a similar eficacia y satisfacción de los pacientes que el tratamiento convencional. Sin embargo, debemos ser cautos antes de recomendar sobre la base de estos resultados el uso de nuevas quinolonas para el manejo de la NAC en pacientes sin riesgo de infección por neumococos resistentes a penicilina.

Dr. Martín 0 'Flaherty Servicio de Clínica Médica. Universidad Austral 